\title{
THE SEMI-SIMPLICITY MANIFOLD OF ARBITRARY OPERATORS
}

\author{
BY \\ SHMUEL KANTOROVITZ(1)
}

Introduction. In finite dimensional complex Euclidian space, any linear operator has a unique maximal invariant subspace on which it is semi-simple. It is easily described by means of the Jordan decomposition theorem. The main result of this paper (Theorem 2.1) is a generalization of this fact to infinite dimensional reflexive Banach space, for arbitrary bounded operators $T$ with real spectrum. Our description of the "semi-simplicity manifold" for $T$ is entirely analytic and basis-free, and seems therefore to be a quite natural candidate for such generalizations to infinite dimension.

A similar generalization to infinite dimension of the concepts of Jordan cells and Weyr characteristic will be presented elsewhere.

The theory is motivated and illustrated by examples in $\S 3$.

Notations. The following notations are fixed throughout this paper, and will be used without further explanation.

$R$ : the field of real numbers.

$C$ : the field of complex numbers.

$\mathscr{B}$ : the sigma-algebra of all Borel subsets of $\boldsymbol{R}$.

$C(R)$ : the space of all continuous complex valued functions on $\boldsymbol{R}$.

$L^{p}(R)$ : the usual Lebesgue spaces on $R(p \geqq 1)$.

$\int$ : integration over $\boldsymbol{R}$.

$\hat{f}$ : the Fourier transform of $f \in L^{1}(R)$.

$X$ : an arbitrary complex Banach space.

$X^{*}$ : the conjugate of $X$.

$B(X)$ : the Banach algebra of all bounded linear operators acting on $X$.

$I$ : the identity operator on $X$.

$|\cdot|$ : the original norms on $X, X^{*}$ and $B(X)$ (new norms, to be defined, will be denoted by double bars).

$|\cdot|_{1},|\cdot|_{\infty}:$ the $L^{1}(R)$ and $L^{\infty}(R)$ norms (respectively).

$\sigma(T)$ : the spectrum of $T \in B(X)$;

$\rho(T):$ the resolvent set of $T$;

$R(\lambda ; T)$ : the resolvent of $T$;

$T \mid W:$ the restriction of $T$ to an invariant linear manifold $W$.

Received by the editors September 27, 1965.

(1) Research supported by U.S. Army Research Office, Durham. 
1. The semi-simplicity manifold. Let $T \in B(X)$ be a fixed operator with real spectrum.

Definition 1.1. For $x \in X$, we let

and

$$
\|x\|=\sup \left\{\left|\int f(t) e^{i t T} x d t\right| ; f \in L^{1}(R),|\hat{f}|_{\infty} \leqq 1\right\} .
$$

$$
W=W(T)=\{x \in X ;\|x\|<\infty\} .
$$

If the integral in the definition of $\|x\|$ does not converge (strongly) for some $f \in L^{1}(R)$, we set $\|x\|=\infty$. Clearly, $W$ contains all the eigenvectors of $T$.

THEOREM 1.1. For any $x \in X$,

$$
\|x\|=\sup \left|\sum_{j=1}^{n} c_{j} \exp \left(i t_{j} T\right) x\right|,
$$

where the sup is taken over all $n=1,2, \cdots, c_{j} \in C$ and $t_{j} \in R(1 \leqq j \leqq n)$ for which $\left|\sum_{j=1}^{n} c_{j} \exp \left(i t_{j} s\right)\right|_{\infty} \leqq 1$.

Proof. Denote the supremum above by $\|x\|^{\prime}$. Suppose $\|x\|<\infty$. For any $x^{*} \in X^{*}$ and $f \in L^{1}(R)$, we have

$$
\left|\int f(t) x^{*} e^{i t} x d t\right| \leqq\|x\|\left|x^{*}\right||\hat{f}|_{\infty}
$$

By Schoenberg's Criterion [9], there exists a finite regular Borel measure $\mu=\mu\left(\cdot \mid x, x^{*}\right)$ on $R$ such that

$$
\|\mu\| \leqq\|x\|\left|x^{*}\right|
$$

and

$$
x^{*} e^{i t T} x=\int e^{i t s} d \mu(s)
$$

Therefore [1]

$$
\left|\sum_{j=1}^{n} c_{j} x^{*} \exp \left(i t_{j} T\right) x\right| \leqq\|x\|\left|x^{*}\right|\left|\sum_{j=1}^{n} c_{j} \exp \left(i t_{j} s\right)\right|_{\infty}
$$

for all $c_{j} \in C, t_{j} \in R$ and $n=1,2, \cdots$. Hence $\|x\|^{\prime} \leqq\|x\|$.

Next, suppose $\|x\|^{\prime}<\infty$.

By Bochner's Criterion [1], there is a representation (1.2) with $\|\mu\| \leqq\|x\|^{\prime}\left|x^{*}\right|$. In particular (or directly from the definition of $\left.\|x\|^{\prime}\right),\left|e^{i T} x\right| \leqq\|x\|^{\prime}$. Therefore the integral $\int f(t) e^{i t} T_{x} d t$ converges strongly for each $f \in L^{1}(R)$, and so

$$
\left|\int f(t) e^{i t T} x d t\right|=\sup _{\left|x^{*}\right|=1}\left|\int f(t) x^{*} e^{i t T} x d t\right| \leqq\|\mu\||\hat{f}|_{\infty} \leqq\|x\|^{\prime}|\hat{f}|_{\infty}
$$

i.e., $\|x\| \leqq\|x\|^{\prime}$. Q.E.D. 
THEOREM 1.2. (a) $(W,\|\cdot\|)$ is a normed linear space, and $\|x\| \geqq|x|$ (for all $x \in X)$. (b) $W$ is an invariant linear manifold for any $S \in B(X)$ which commutes with $T$, and $S$ is continuous on $(W,\|\cdot\|)$ with bound $\leqq|S|$.

Proof. (a) Clearly, $\|x+y\| \leqq\|x\|+\|y\|$ and $\|\lambda x\|=|\lambda|\|x\| \quad(x, y \in W$; $\lambda \in C$ ). Therefore $W$ is a linear manifold. Taking $n=1, c_{1}=1$ and $t_{1}=0$, we have $\left|\sum_{j=1}^{n} c_{j} \exp \left(i t_{j} s\right)\right|_{\infty}=1$ and $\left|\sum_{j=1}^{n} c_{j} \exp \left(i t_{j} T\right) x\right|=|x|$, hence $|x| \leqq\|x\|$ (by Theorem 1.1). In particular, $\|\cdot\|$ is a norm on $W$, and (a) is proved.

(b) Let $S \in B(X)$ commute with $T$, and let $x \in W$. Suppose $c_{j} \in C$ and $t_{j} \in R$ $(j=1, \cdots, n)$ are such that $\left|\Sigma_{j=1}^{n} c_{j} \exp \left(i t_{j} s\right)\right|_{\infty} \leqq 1$. Then, by Theorem 1.1,

$$
\left|\sum_{j=1}^{n} c_{j} \exp \left(i t_{j} T\right) S x\right|=\left|S \sum_{j=1}^{n} c_{j} \exp \left(i t_{j} T\right) x\right| \leqq|S|\|x\|,
$$

i.e., $S x \in W$ and $\|S x\| \leqq|S|\|x\|$, proving (b).

Definition 1.2. The semi-simplicity manifold for $T$ is the linear manifold $W=W(T)$.

REMARK. The norms $\|\cdot\|$ and $|\cdot|$ are equivalent (or, what amounts to the same thing, $W=X$ ) if and only if $T$ is of class $C$ (i.e., if and only if $T$ is scalar for $X$ weakly complete, or $T$ is similar to a hermitian operator if $X$ is a Hilbert space; cf. [7]). In particular, if $X$ is a Hilbert space, $\|\cdot\|=|\cdot|$ if and only if $T$ is hermitian (cf. Theorem 3.4 in [7]).

An equivalent definition of $W$ follows from our next expression for the nor $m$ $\|x\|$.

TheOREM 1.3. For any $x \in X$,

$$
\|x\|=\sup \left\{\frac{1}{2 \pi} \int\left|x^{*}[R(s-i \varepsilon ; T)-R(s+i \varepsilon ; T)] x\right| d s\right\}
$$

where the sup is taken over all $\varepsilon>0$ and all unit vectors $x^{*} \in X^{*}$.

Proof. Let $\varepsilon>0$ and

$$
F_{e}(s)=\frac{1}{2 \pi i}[R(s-i \varepsilon ; T)-R(s+i \varepsilon ; T)], \quad s \in R .
$$

Since $\sigma(T)$ is real, $F_{\varepsilon}$ is well defined and $x^{*} F_{\varepsilon}(\cdot) x \in L^{1}(R) \cap L^{2}(R)$ for any $x \in X$ and $x^{*} \in X^{*}$. By $\left[6\right.$, p. 164, equation (2)], the Fourier transform of $x^{*} F_{\varepsilon}(\cdot) x$ is equal to $\exp (-2 \pi \varepsilon|t|) x^{*} e^{-2 \pi i t} T$. But $\exp (-2 \pi \varepsilon|t|)$ is the Fourier transform of $f_{\varepsilon}(s)=\varepsilon\left(s^{2}+\varepsilon^{2}\right)^{-1} / \pi$, and if $x \in W, x^{*} \exp (-2 \pi i t T) x$ is the FourierStieltjes transform of the measure $\mu$ defined in (1.1) and (1.2). Thus $x^{*} F_{\varepsilon}(\cdot) x$ is the convolution of $f_{\varepsilon} \in L^{1}(R)$ and $\mu$, and therefore, using (1.1):

$$
\left|x^{*} F_{\varepsilon}(\cdot) x\right|_{1} \leqq\left|f_{\varepsilon}\right|_{1}\|\mu\|=\|\mu\| \leqq\|x\|\left|x^{*}\right| .
$$


Denoting the supremum in the statement of the theorem by $\|x\|$ ", we have proved that $\|x\|^{\prime \prime} \leqq\|x\|$ whenever $\|x\|<\infty$.

Next, suppose $\|x\|^{\prime \prime}<\infty$ for some $x \in X$. Then, for $\varepsilon>0$ and $x^{*} \in X^{*}$ with unit norm,

$$
\begin{aligned}
\left|\exp (-2 \pi \varepsilon|t|) x^{*} \exp (-2 \pi i t T) x\right| & =\left|\left[x^{*} F_{\varepsilon}(\cdot) x\right]^{\wedge}\right| \leqq\left|x^{*} F_{\varepsilon}(\cdot) x\right|_{1} \\
& \leqq\|x\|^{\prime \prime}
\end{aligned}
$$

i.e., $\exp (-2 \pi \varepsilon|t|)|\exp (-2 \pi i t T) x| \leqq\|x\|^{\prime \prime}$ (for all $t \in \boldsymbol{R}$ ). Therefore the integral $\int f(t) \exp (-2 \pi \varepsilon|t|) \exp (-2 \pi i t T) x d t$ converges strongly for any $f \in L^{1}(R)$, and the dominated convergence theorem implies that

$$
\int f(t) \exp (-2 \pi i t T) x d t
$$

(converges strongly and) is equal to

$$
\lim _{\varepsilon \rightarrow 0+} \int f(t) \exp (-2 \pi \varepsilon|t|) \exp (-2 \pi i t T) x d t
$$

(strongly). Since $\exp (-2 \pi \varepsilon|t|) x^{*} \exp (-2 \pi i t T) x$ is the Fourier transform of $x^{*} F_{\varepsilon}(\cdot) x$ and $\left|x^{*} F_{\varepsilon}(\cdot) x\right|_{1} \leqq\|x\|^{\prime \prime}$, the latter integral has norm $\leqq\|x\|^{\prime \prime}|\hat{f}|_{\infty}$ (for all $\varepsilon>0$ and $f \in L^{1}(R)$ ). Hence $\|x\| \leqq\|x\|$ ". Q.E.D.

2. Spectral decomposition of $T$ on $W$. If $Z$ is any linear manifold in $X$, we note by $T(Z)$ the algebra of all linear transformations of $X$ with domain $Z$ and range contained in $Z$.

Definition 2.1. Let $Z$ be a linear manifold in $X$. A spectral measure on $Z$ is a map $\delta \rightarrow E(\delta)$ of $\mathscr{B}$ into $T(Z)$ such that

(i) $E(R)=I \mid Z$;

(ii) $E(\delta) E(\varepsilon)=E(\delta \cap \varepsilon)$ for all $\delta, \varepsilon \in \mathscr{B}$; and

(iii) for each $x \in Z, E(\cdot) x$ is a regular strongly countably additive vector measure on $\mathscr{B}$.

By Corollary III.4.5 in [3], $E(\cdot) x$ is necessarily bounded (with a bound depending of course on $x \in Z$ ).

If $Z$ is of the second category in $X$ and if each $E(\delta)$ is closed, the closed graph and the uniform boundedness theorems imply that $E(\delta) \in B(X)$ (all $\delta$ ) and $\sup _{\delta}|E(\delta)|<\infty$, so that our definition coincides in this case with the usual one.

We can state now the main result of this paper.

THEOREM 2.1. Let $X$ be reflexive, and $T \in B(X)$ have real spectrum. Let $W$ be the semi-simplicity manifold for $T$. Then, for all $x \in W$ and all polynomials $p$,

$$
p(T) x=\int p(t) d E(t) x,
$$


where $E$ is a spectral measure on $W$ supported by $\sigma(T)$ and commuting with each $S \in B(X)$ which commutes with $T$. The representation (2.1) is "maximalunique" in the following sense: if $W^{\prime}$ is an invariant linear manifold for $T$, and (2.1) is valid with $W^{\prime}$ and $E^{\prime}$ replacing $W$ and $E$ respectively, then $W^{\prime} \subset W$ and $E^{\prime}(\delta)=E(\delta) \mid W^{\prime}$ for all $\delta \in \mathscr{B}$.

Proof. Assuming that representations (2.1) exist with spectral measures $E$ and $E^{\prime}$ on $W$ and $W^{\prime}$ respectively, both supported by $\sigma(T)$, we show first that $W^{\prime} \subset W$ and $E^{\prime}(\delta)=E(\delta) \mid W^{\prime}$ for all $\delta \in \mathscr{B}$. Fix $x \in W^{\prime}$. Since $p(T) x=\int_{\sigma(T)} p(t) d E^{\prime}(t) x$ for any polynomial $p$, and since $\sigma(T)$ is compact, we obtain

$$
\left.e^{i t} T_{x}=\int_{\sigma(T)} e^{i t s} d E^{\prime}(s) x \quad \text { (all } t \in R\right) .
$$

Let $M(x)$ be a uniform bound for the vector measure $E^{\prime}(\cdot) x$. Then, for $c_{j} \in C$ and $t_{j} \in R(j=1, \cdots, n)$, we have

$$
\begin{aligned}
\left|\sum_{j=1}^{n} c_{j} \exp \left(i t_{j} T\right) x\right| & =\left|\int_{\sigma(T)}\left(\sum_{j=1}^{n} c_{j} \exp \left(i t_{j} s\right)\right) d E^{\prime}(s) x\right| \\
& \leqq 4 M(x)\left|\sum_{j=1}^{n} i c_{j} \exp \left(i t_{j} s\right)\right|_{\infty},
\end{aligned}
$$

i.e., $\|x\| \leqq 4 M(x)$ (by Theorem 1.1) and $x \in W$. Thus $W^{\prime} \subset W$. For $x \in W^{\prime}$, equation (2.2) is valid with both $E$ and $E^{\prime}$; i.e., $\int e^{i t s} d E(t) x=\int e^{i t s} d E^{\prime}(t) x$ for all $t \in \boldsymbol{R}$. Hence $E(\delta) x=E^{\prime}(\delta) x$ for all $\delta \in \mathscr{B}$, by the uniqueness of the Fourier transform. This proves the maximality-uniqueness assertion.

The existence of the spectral decomposition (2.1) on the semi-simplicity manifold $W$ will be proved in the following sequence of eight lemmas.

LEMMA 2.1. There exists a family $\{E(\delta) ; \delta \in \mathscr{B}\}$ of linear transformations of $X$ with domain $W$ with the following properties:

(i) $E(\boldsymbol{R})=I \mid W$;

(ii) for each $x \in W, E(\cdot) x$ is a regular strongly countably additive vector measure on $\mathscr{B}$; and

(iii) $e^{i t T} x=\int e^{i t s} d E(s) x$ for all $t \in R$ and $x \in W$.

Proof. Fix $x \in W$ and $x^{*} \in X^{*}$. Let $\mu=\mu\left(\cdot \mid x, x^{*}\right)$ be as in (1.1) and (1.2). The uniqueness of the representation (1.2) implies that $\mu\left(\delta \mid x, x^{*}\right)$ is a continuous linear functional on $X^{*}$ (with bound $\leqq\|x\|$ ) for each $\delta \in \mathscr{B}$ and $x \in W$ fixed. Since $X$ is reflexive, there exists a unique element of $X$, which we denote by $E(\delta) x$, such that $|E(\delta) x| \leqq\|x\|$ and $\mu\left(\delta \mid x, x^{*}\right)=x^{*} E(\delta) x$ for all $x^{*} \in X^{*}$. Similarly, for $\delta$ and $x^{*}$ fixed, $\mu\left(\delta \mid x, x^{*}\right)$ is a linear function of $x$ on $W$; therefore the map $x \rightarrow E(\delta) x$ is a linear transformation of $X$ with domain $W$. Now, (i) follows from (1.2) with $t=0$; (ii) follows from the equation $x^{*} E(\cdot) x=\mu\left(\cdot \mid x, x^{*}\right)$ and Theorem IV.10.1 in [3]; (iii) is a rewriting of (1.2), which is justified by (ii). Q.E.D. 
LEMMA 2.2. Let $\{E(\delta)\}$ be as in Lemma 2.1. Then $E(\delta)$ commutes with each $S \in B(X)$ which commutes with $T$ (for all $\delta \in \mathscr{B})$.

Proof. If $S \in B(X)$ commutes with $T$, then $S W \subset W$ by Theorem 1.2. Therefore, for each $x \in W$,

$$
e^{i t} S x=\int e^{i t s} d E(s) S x
$$

The left-hand side is equal to $S e^{i t} T$, which, by Lemma 2.1 (iii) and (ii), is given by $\int e^{i t s} d S E(s) x$. The lemma follows now from the uniqueness of the Fourier transform.

Next, we show that the vector measures $E(\cdot) x(x \in W)$ have compact support (this will be refined in Lemma 2.6).

LEMMA 2.3. The supports of the vector measures $E(\cdot) x$ are contained in $[-|T|,|T|]$ (for all $x \in W)$.

Proof. Fix $x \in W$ and $x^{*} \in X^{*}$, and let $\mu=x^{*} E(\cdot) x$. The function $f(z)=x^{*} e^{i z T} x$ of the complex variable $z$ is entire of exponential type $\leqq|T|$. For $t \in R, f(t)=O(1)$ (in fact, $|f(t)| \leqq\|x\|\left|x^{*}\right|$ by (1.1) and (1.2)). Consider $f$ as a distribution on the Schwartz space $\mathscr{S}$ of rapidly decreasing functions on $\boldsymbol{R}$. By Schwartz' generalization of the Paley-Wiener theorem (cf. [10] or [5, Theorem 5 , p. 145]), the Fourier transform $\hat{f}$ of the distribution $f$ has its support in the interval $|t| \leqq|T|$. Since $\hat{f}=2 \pi \mu$ (by 1.2 ), the lemma is proved.

Notation. Let $\boldsymbol{B}(\Delta)$ denote the space of all complex Borel functions $f$ on $\boldsymbol{R}$, which are bounded on $\Delta=[-|T|,|T|]$, with the pseudo-norm $|f|_{\Delta}=\sup _{\Delta}|f|$.

LEMMA 2.4. The map

$$
\pi:(f, x) \rightarrow \int f(s) d E(s) x
$$

of $\boldsymbol{B}(\Delta) \times W$ into $X$ is continuous, has range in $W$, and is also continuous as a map of $\boldsymbol{B}(\Delta) \times W$ into $(W,\|\cdot\|)$. In fact,

$$
\left\|\int f(s) d E(s) x\right\| \leqq\|x\||f|_{\Delta}
$$

for all $f \in \boldsymbol{B}(\Delta)$ and $x \in W$.

Proof. Fix $x \in W$. Let

$$
h(s)=\sum_{j=1}^{n} c_{j} \exp \left(i t_{j} s\right) \quad\left(c_{j} \in C ; t_{j} \in R ; j=1, \cdots, n\right)
$$

and write 


$$
H=\sum_{j=1}^{n} c_{j} \exp \left(\mathrm{it}_{j} T\right)
$$

Since $H \in B(X)$ commutes with $T$, we have $H x \in W$ (Theorem 1.2). Let us estimate $\|H x\|$. In what follows, all suprema are taken over all $g \in L^{1}(R)$ with $|\hat{g}|_{\infty} \leqq 1$. We have:

$$
\begin{aligned}
\|H x\| & =\sup \left|\int g(t) e^{i t} T_{H x d t}\right| \\
& =\sup \left|\sum_{j=1}^{n} c_{j} \int g(t) \exp \left[i\left(t+t_{j}\right) T\right] x d t\right| \\
& =\sup \left|\int\left[\sum_{j=1}^{n} c_{j} g\left(t-t_{j}\right)\right] e^{i t} T_{x} d t\right| .
\end{aligned}
$$

Since $\sum_{j=1}^{n} c_{j} g\left(t-t_{j}\right)=g^{*}(t) \in L^{1}(R)$ and $x \in W$, we obtain

$$
\|H x\| \leqq \sup \left|\hat{g}^{*}\right|_{\infty}\|x\| \quad \text { (sup over } g \text {, as before). }
$$

But $\hat{g}^{*}(s)=\sum_{j=1}^{n} c_{j} \exp \left(i t_{j} s\right) \hat{g}(s)=h(s) \hat{g}(s)$, so that $\left|\hat{g}^{*}\right|_{\infty} \leqq|h|_{\infty}|\hat{g}|_{\infty} \leqq|h|_{\infty}$; hence

$$
\|H x\| \leqq|h|_{\infty}\|x\| .
$$

Now, for $x \in W$ and $f \in \boldsymbol{B}(\Delta)$ fixed, let $y=\pi(f, x)=\int f(s) d E(s) x$. By Lemma 2.1 (ii), this is a well-defined element of $X$, and for $H$ as above and $x^{*} \in X^{*}$ with unit norm, we have (using Lemmas 2.2 and 2.3):

$$
\begin{aligned}
\left|x^{*} H y\right| & =\left|\int_{\Delta} f(s) d x^{*} H E(s) x\right|=\left|\int_{\Delta} f(s) d x^{*} E(s) H x\right| \\
& =\left|\int_{\Delta} f(s) d \mu\left(s \mid H x, x^{*}\right)\right| \\
& \leqq|f|_{\Delta}\left\|\mu\left(\cdot \mid H x, x^{*}\right)\right\| \leqq|f|_{\Delta}\|H x\| \\
& \leqq|f|_{\Delta}|h|_{\infty}\|x\|,
\end{aligned}
$$

where we have written $\mu\left(\cdot \mid x, x^{*}\right)=x^{*} E(\cdot) x$ and applied (1.1) and (2.4). Hence $|H y| \leqq|f|_{\Delta}\|x\||h|_{\infty}$, and therefore, by Theorem 1.1, $\|y\| \leqq|f|_{\Delta}\|x\|$. This proves (2.3) and the lemma. Taking in particular $f=c_{\delta}$ (the characteristic function of $\delta \in \mathscr{B})$, we obtain

COROllaRy 2.1. For each $\delta \in \mathscr{B}, E(\delta) \in T(W)$ and $\|E(\delta) x\| \leqq\|x\|$ (for all $x \in W)$.

Notation. We write $T(f) x=\pi(f, x)=\int f(s) d E(s) x$.

By Lemma 2.4, $T(f) \in T(W)$ and 


$$
\|T(f) x\| \leqq|f|_{\Delta}\|x\| \text { for all } f \in B(\Delta) \text { and } x \in W .
$$

By Lemma 2.2, $T(f)$ commutes with each $S \in B(X)$ which commutes with $T$.

The next lemma follows from a standard density argument, which we reproduce because of the special precautions needed in dealing with our unbounded operators.

Lemma 2.5. The map $f \rightarrow T(f)$ of $C(R)$ into $T(W)$ is multiplicative, and $T(p)=p(T) \mid W$ for all polynomials $p$.

Proof. By Lemmas 2.1 and 2.3,

$$
\left.e^{i t T} x=\int_{\Delta} e^{i t s} d E(s) x \quad \text { (all } x \in W \text { and } t \in R\right) .
$$

Expanding both sides in powers of $t$, we obtain for $x \in W, T^{n} x=\int_{\Delta} s^{n} d E(s) x$ $(n=0,1,2, \cdots)$, and therefore $T(p)=p(T) \mid W$ for all polynomials $p$. It follows in particular that the map $f \rightarrow T(f)$ of $\boldsymbol{B}(\Delta)$ into $\boldsymbol{T}(W)$ is multiplicative when restricted to polynomials. If $f, g \in C(R)$, choose polynomials $p_{n}, q_{n}(n=1,2, \cdots)$ such that $p_{n} \rightarrow f$ and $q_{n} \rightarrow g$ uniformly on $\Delta$. Fix $x \in W$. By Theorem 1.2,

$$
\begin{aligned}
|[T(f) T(g)-T(f g)] x| \leqq & \left\|T\left(f-p_{n}\right) T(g) x\right\| \\
& +\left\|T\left(p_{n}\right) T\left(g-q_{n}\right) x\right\|+\left\|T\left(p_{n} q_{n}-f g\right) x\right\| \\
& =A+B+C .
\end{aligned}
$$

By (2.5),

$$
A \leqq\left|f-p_{n}\right|_{\Delta}\|T(g) x\| \leqq\left|f-p_{n}\right|_{\Delta}|g|_{\Delta}\|x\| .
$$

Since $T\left(p_{n}\right)=p_{n}(T) \mid W$ commutes with $T(h)$ for any $h \in B(\Delta)$, we have by (2.5):

$$
\begin{aligned}
B & =\left\|T\left(g-q_{n}\right) T\left(p_{n}\right) x\right\| \leqq\left|g-q_{n}\right|_{\Delta}\left\|T\left(p_{n}\right) x\right\| \\
& \leqq\left.\left.\left|g-q_{n}\right|_{\Delta}\right|_{n}\right|_{\Delta}\|x\| .
\end{aligned}
$$

Finally,

$$
C \leqq\left|p_{n} q_{n}-f g\right|_{\Delta}\|x\|
$$

Letting $n \rightarrow \infty$, we obtain $T(f) T(g)=T(f g)$. Q.E.D.

LEMMA 2.6. The support of $E(\cdot) x$ lies in $\sigma(T)$ (for each $x \in W$ ).

Proof. (Foias [4], proof of Proposition 1.) Suppose $f \in C(R)$ has compact support disjoint from $\sigma(T)$. From $z \in C \mid \operatorname{supp} f$, define $f_{z}(t)=(z-t)^{-1} f(t)$. Clearly, $f_{z} \in C(R)$ and $T\left(f_{z}\right) x$ is an analytic $X$-valued function of $z$ on $C \backslash$ supp $f$, for each $x \in W$. By Lemma 2.5 ,

$$
(z I-T) T\left(f_{z}\right) x=T(z-t) T\left(f_{z}\right) x=T\left((z-t) f_{z}\right) x=T(f) x \quad(\text { all } x \in W) .
$$

Thus, for $z \in(\boldsymbol{C} \backslash \operatorname{supp} f) \cap \rho(T)$,

$$
T\left(f_{z}\right) x=R(z ; T) T(f) x \quad(\text { all } x \in W),
$$


and the right-hand side provides an analytic continuation of $T\left(f_{z}\right) x$ to $\rho(T) \supset \operatorname{supp} f$. Hence $T\left(f_{z}\right) x$ is entire, and since $T\left(f_{z}\right) x \rightarrow 0$ for $z \rightarrow \infty$ (by (2.6)), we have $T\left(f_{z}\right) x=0$ and hence $T(f) x=0$ (by (2.6)) for all $x \in W$. This proves that for each $x \in W$ and $x^{*} \in X^{*}$, the support of the linear functional on $C(R)$ defined by $f \rightarrow \int f(t) d x^{*} E(t) x$ is contained in $\sigma(T)$. Equivalently, the supports of the complex measures $x^{*} E(\cdot) x$ (and so, of the vector measures $E(\cdot) x$ ) are contained in $\sigma(T)$ (for all $x \in W$ and $x^{*} \in X^{*}$ ).

LEMMA 2.7. If $f \in C(\boldsymbol{R})$ and $g \in \boldsymbol{B}(\Delta)$, then $T(f)$ and $T(g)$ commute (as elements of $T(W))$. In particular, $E(\delta)$ commutes with $T(f)$ for all $\delta \in \mathscr{B}$ and $f \in C(R)$.

Proof. Choose polynomials $p_{n}$ which converge uniformly to $f$ on $\Delta$. Since $T(g)$ commutes with $p_{n}(T)=T\left(p_{n}\right)$ (by Lemma 2.2), we obtain (by (2.5) and Theorem 1.2):

$$
\begin{aligned}
& |[T(g) T(f)-T(f) T(g)] x| \leqq\left\|T(g) T\left(f-p_{n}\right) x\right\|+\left\|T\left(f-p_{n}\right) T(g) x\right\| \\
& \leqq|g|_{\Delta}\left\|T\left(f-p_{n}\right) x\right\|+\left|f-p_{n}\right|_{\Delta}\|T(g) x\| \\
& \leqq 2\left|f-p_{n}\right|_{\Delta}|g|_{\Delta}\|x\| \rightarrow 0 \\
& n \rightarrow \infty
\end{aligned}
$$

for all $x \in W$, Q.E.D.

LeMma 2.8. $E(\delta \cap \varepsilon)=E(\delta) E(\varepsilon)$ for all $\delta, \varepsilon$ in $\mathscr{B}$.

Proof. Fix $\delta \in \mathscr{B}, x \in W$ and $g \in C(\boldsymbol{R})$. Recalling that $T(g) x \in W$ (Lemma 2.4), we may choose a finite positive measure $\lambda$ such that the vector measures $E(\cdot) x$ and $E(\cdot) T(g) x$ are both $\lambda$-continuous (cf. $\left[3\right.$, p. 321]). Let $c_{\delta}$ denote the characteristic function of $\delta$, and choose $f_{n} \in C(\boldsymbol{R})$ such that $\left|f_{n}\right| \leqq 1$ and $f_{n} \rightarrow c_{\delta}$ a.e.

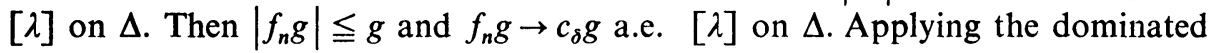
convergence theorem for vector measures (cf. [3, p. 328]), we obtain that $\lim _{n \rightarrow \infty} T\left(f_{n}\right) T(g) x=E(\delta) T(g) x$ and $\lim _{n \rightarrow \infty} T\left(f_{n} g\right) x=T\left(c_{\delta} g\right) x$. However, by Lemma 2.5, $T\left(f_{n}\right) T(g) x=T\left(f_{n} g\right) x$. Hence $T\left(c_{\delta} g\right)=E(\delta) T(g)$, and thus, by Lemma 2.7, $T\left(c_{\delta} g\right)=T(g) E(\delta)$. Equivalently,

$$
\int g(t) c_{\delta}(t) d E(t) x=\int g(t) d E(t) E(\delta) x
$$

for all $g \in C(\boldsymbol{R})$ and $x \in W$ (since $g$ and $x$ were arbitrary). The uniqueness of the Riesz representation implies that $\int_{\varepsilon} c_{\delta}(t) d E(t) x=\int_{\varepsilon} d E(t) E(\delta) x$ for each $\varepsilon$ in $\mathscr{B}$ and $x$ in $W$. Equivalently, $E(\varepsilon \cap \delta) x=E(\varepsilon) E(\delta) x$ for all $x \in W$ and $\varepsilon, \delta \in \mathscr{B}$. Q.E.D.

This completes the proof of Theorem 2.1. If we replace $W$ by its completion with respect to $\|\cdot\|$, the structure of $T$ can be described in previously established terminology. This has however the inconvenience of taking us out from the original space $X$ (in general). 
DefinItION 2.2. The semi-simplicity space for $T$ is the completion $Y$ of the normed linear space $(W,\|\cdot\|)$.

By Theorem 1.2, if $S \in B(X)$ commutes with $T$, then $S \mid W$ has a unique extension $S_{Y}$ as a bounded linear operator on $Y$, and $\left\|S_{Y}\right\| \leqq|S|$ (norms in $Y, Y^{*}$ and $B(Y)$ are denoted by double bars). In the following corollary, we use the the terminology of [2] and [7].

COROLlaRY 2.2. Let $X$ be an arbitrary Banach space (not necessarily reflexive), and let $T \in B(X)$ have real spectrum. Let $Y$ be the semi-simplicity space for $T$. Then $T_{Y}$ is of class $C$. (Thus $T_{Y}{ }^{*}$ is spectral of class $Y$ and scalar type, and if $Y$ is weakly complete, $T_{Y}$ is spectral of scalar type.)

Proof. By (2.5) and Lemma 2.5, $\|p(T) x\| \leqq|p|_{\Delta}\|x\|$ for all polynomials $p$ and $x \in W$. Hence $\left\|p(T)_{Y}\right\| \leqq|p|_{\Delta}$, i.e., $\left\|p\left(T_{Y}\right)\right\| \leqq \sup _{\Delta}|p|$ for all polynomials p. Q.E.D.

Another description of the semi-simplicity manifold of an operator with real spectrum follows from Theorem 2.1. Suppose, without loss of generality, that $\sigma(T) \subset[0,1]$. Then

\section{COROllary 2.3 .}

$$
\|x\|=\sup \left\{\sum_{j=0}^{n}\left(\begin{array}{l}
n \\
j
\end{array}\right)\left|x^{*} T^{j}(I-T)^{n-j} x\right| ;\left|x^{*}\right|=1, \quad n=0,1,2, \cdots\right\} .
$$

Proof. Denote the right-hand side by $\|x\|^{\prime}$. Suppose $\|x\|<\infty$. Then, by Theorem 2.1 (Equation (2.1)):

$$
\begin{aligned}
\sum_{j=0}^{n}\left(\begin{array}{c}
n \\
j
\end{array}\right)\left|x^{*} T^{j}(I-T)^{n-j} x\right|=\sum_{j=0}^{n}\left(\begin{array}{c}
n \\
j
\end{array}\right)\left|\int_{0}^{1} s^{j}(1-s)^{n-j} d x^{*} E(s) x\right| \\
\leqq \sum_{j=0}^{n} \int_{0}^{1}\left(\begin{array}{c}
n \\
j
\end{array}\right) s^{j}(1-s)^{n-j} d u\left(s \mid x, x^{*}\right) \\
=\int_{0}^{1} d u\left(s \mid x, x^{*}\right)=\left\|\mu\left(\cdot \mid x, x^{*}\right)\right\| \leqq\|x\|\left|x^{*}\right|
\end{aligned}
$$

where $u$ is the variation of the complex measure $\mu=x^{*} E(\cdot) x$ (cf. (1.1)). Thus $\|x\|^{\prime} \leqq\|x\|$. The reversed inequality follows at once from the Hausdorff moments theorem, as in the proof of Theorem 2.18 in [8].

\section{Examples.}

3.1. Suppose $X$ is finite dimensional. Let $x_{1}, \cdots, x_{n}$ be a basis of $X$ with respect to which $T$ has a Jordan canonical matrix representation; i.e., for suitable integers $0=n_{0}<n_{1}<n_{2}<\cdots<n_{k}=n$ and a suitable enumeration $\lambda_{1}, \cdots, \lambda_{k}$ of $\sigma(T)$ (with possible repetitions), $T x_{q}=\lambda_{j} x_{q}+x_{q+1}$ for $n_{j-1}<q<n_{j}$ and $T x_{n_{j}}=\lambda_{j} x_{n_{j}}$ $(j=1, \cdots, k)$. Since $e^{i t} x_{n_{j}}=\exp \left(i t \lambda_{j}\right) x_{n_{j}}$ and $\lambda_{j}$ are real (our standing assumption 
about $\sigma(T)$ !), it follows trivially that $x_{n_{j}} \in W(T)(j=1, \cdots, k)$. For $n_{j-1}<q<n_{j}$, we have $\left(T-\lambda_{j} I\right)^{n_{j}-q+1} x_{q}=0$ and $\left(T-\lambda_{j} I\right)^{n_{j}-q} x_{q} \neq 0$. Therefore

$$
\begin{aligned}
\left|e^{i t T} x_{q}\right| & =\left|e^{i t\left(T-\lambda_{j} I\right)} x_{q}\right| \\
& =\left|\sum_{m=0}^{n_{j}-q} \frac{(i t)^{m}}{m !}\left(T-\lambda_{j} I\right)^{m} x_{q}\right| \neq O(1)
\end{aligned}
$$

when $|t| \rightarrow \infty$, because $n_{j}-q \geqq 1$. Thus $x_{q} \notin W(T)$ for $n_{j-1}<q<n_{j}$.

This shows that $W(T)$ is the linear span of $x_{n_{1}}, \cdots, x_{n_{k}}$; its dimension is exactly $k$, the number of Jordan cells. Of course, our definition of $W(T)$ does not depend on a choice of basis.

3.2. Let $X=L^{p}[0,1](1 \leqq p<\infty)$ and

$$
(T \phi)(x)=x \phi(x)+\int_{x}^{1} \phi(s) d s, \quad \phi \in L^{p}[0,1], x \in[0,1] .
$$

Then, for $t \in R$,

$$
\left(e^{i t T} \phi\right)(x)=e^{i t x} \phi(x)+i t \int_{x}^{1} e^{i t s} \phi(s) d s .
$$

The characteristic function $c_{s}$ of the interval $[0, s](0<s \leqq 1)$ is an eigenvector corresponding to the eigenvalue $s$. Since the functions $c_{s}(0 \leqq s \leqq 1)$ are trivially in $W(T)$ and are dense in $X, W(T)$ is dense in $X$. A spectral decomposition of $T$ on the linear span $W_{0}$ of $c_{s}(0 \leqq s \leqq 1)$ is very easily described (cf. last example in [8]). However $W(T)$ is much wider than $W_{0}$. Indeed, suppose $\phi$ is a function of bounded variation in $[0,1]$. Assume, without loss of generality, that $\phi$ is continuous from the left. An integration by parts shows that

$$
\left(e^{i t T} \phi\right)(x)=e^{i t} \phi(1)-\int_{x}^{1} e^{i t s} d \phi(s) .
$$

It is clear from (3) that $\phi \in W(T)$, and so $W(T) \supset B V[0,1]$ (functions and equivalence classes in $L^{p}[0,1]$ are confused as usual).

On the other hand, $W(T) \neq L^{p}[0,1]$. In fact, there are continuous functions which are not in $W(T)$. This follows from (2) and the well-known fact that there exist $2 \pi$-periodic continuous functions with Fourier coefficients which are not $O\left(n^{-1}\right)$. The spectral decomposition of $T$ on $B V[0,1]$ follows easily from (3). The spectral measure on $B V[0,1]$ is given by

$$
(E(\delta) \phi)(x)=c_{\delta}(1) \phi(1)-\int_{\delta \cap[x, 1]} d \phi \quad(\phi \in B V[0,1] ; x \in[0,1] ; \delta \in \mathscr{B}) .
$$

Note that if we consider $T$ as an operator in $B V[0,1]$ (normed as usual by the total variation norm), then $T$ is of class $C$ (i.e., $W(T)$ is the whole space).

If we consider $T$ as an operator on $C[0,1]$, it has no eigenvectors, but still 
$W(T)$ is dense in $C[0,1]$ (and properly contained in it), because $W(T)$ contains all the continuous functions of bounded variation on $[0,1]$.

3.3. The theory has an obvious generalization to closed densely defined operators $T$ such that $i T$ generates a strongly continuous group of operators $T(\cdot)$ on the real line. The norm $\|\cdot\|$ and the manifold $W$ are defined as in Definition 1.1, with $T(t)$ replacing $e^{i t T}$. Consider for example the translation group in $L^{p}(\boldsymbol{R})$ $(1 \leqq p<\infty)$, i.e.,

Then

$$
[T(t) x](s)=x(s-t), \quad x \in L^{p}(R) .
$$

$$
\begin{aligned}
\|x\| & =\sup \left\{\left|\int f(t) x(s-t) d t\right|_{p} ; f \in L^{1}(R),|\hat{f}|_{\infty} \leqq 1\right\} \\
& =\sup \left\{|f * x|_{p} ; f \in L^{1},|\hat{f}|_{\infty} \leqq 1\right\} .
\end{aligned}
$$

This is just the norm $\|\cdot\|_{0}$ considered in [11], and $W$ is the space $\left(L^{p}\right)_{0}$ discussed in this paper in the context of the multipliers problem for Fourier transforms. It is proved there that $W=\{0\}$ for $p<2$, is the whole space for $p=2$, and is dense in $L^{p}$ for $p>2$ ( $W$ contains all the Fourier Transforms of elements of $\left.L^{q}, p^{-1}+q^{-1}=1\right)$. The connection of our work with [1] was noticed by the referee.

\section{REFERENCES}

1 S. Bochner, A theorem on Fourier-Stieltjes integrals, Bull. Amer. Math. Soc. 40 (1934), 271-276.

2. N. Dunford, A survey of the theory of spectral operators, Bull. Amer. Math. Soc. 64 (1958), 217-274.

3. N. Dunford and J. Schwartz, Linear operators, Vol. I, Interscience, New York, 1958.

4. C. Foias, Une application des distributions vectorielles à la théorie spectrale, Bull. Sci. Math. 84(1960), 147-158.

5. A Friedman, Generalized functions and partial differential equations, Prentice-Hall, Englewood Cliffs, N. J., 1963.

6. S. Kantorovitz, On the characterization of spectral operators, Trans. Amer. Math. Soc. 111 (1964), 152-181.

7. - Classification of operators by means of their operational calculus, Trans. Amer. Math. Soc. 115 (1965), 194-224.

8. —_ A Jordan decomposition for operators in Banach space, Trans. Amer. Math. Soc. 122 (1965), 526-550.

9. I. J. Schoenberg, A remark on the preceding note by Bochner, Bull. Amer. Math. Soc. 40 (1943), 277-278.

10. L. Schwartz, Théorie des distributions. II, Actualités Sci. Ind. No. 1122, Hermann, Paris, 1951.

11. A. Figà-Talamanca, On the subspace of $L^{p}$ invariant under multiplication of transform by bounded continuous functions, Rend. Sem. Mat. Univ. Padova 35 (1965), 176-187.

Yale University,

New Haven, Connecticut 